and $\mathrm{O}$ II images has been actually attained, as there are marked differences of detail as well as of distribution in the two images. Take, for example, the long scimitar-like projection on the right-hand side of each photograph with its. companion, or the star, indicated by a cross in Fig. 1, on the outskirts of the nebula, which is surrounded with hydrogen nebulosity, but is clear of O II.

It has already been remarked that the stars involved in the central regions of the Orion nebula are $\mathrm{B}$ type stars, and the radiations $\lambda \lambda 3727,9$ exceptionally strong. As a matter of fact, these radiations are either very faint or absent altogether in many of the planetary nebulæ, where the stars are almost invariably $O$ type of much higher temperatures, although a radiation of shorter wave-length at $\lambda 3426$ is often present. A conspicuous radiation at $\lambda 3869$, as yet unidentified, sometimes appears in place of $\lambda 3727$, although both are present in a few cases.

A comparison of the intensities of $\lambda 5007$ (O III) with $\lambda 3727$ (O II) in the list of planetaries given by Wright (loc. cit.) brings out certain features of interest.

- The spectrum of N.G.C. 40 is unique, for although the slitless quartz spectrograph shows a conspicuous image at $\lambda 3727$ there is no image at $\lambda 5007$, but there is a trace of this radiation in the slit spectrograph exposure.

In three other cases (I.C. 418, N.G.C. 6720, and B.D. $\left.30^{\circ} 3639\right) \lambda 3727$ is brighter than $\lambda 5007$. In ten instances $\lambda 3727$ is absent altogether, with a trace only in two cases, altogether about 25 per cent. of the whole. In nine objects also $\lambda 5007$ is ten times the intensity of $\lambda 3727$, so it appears that the conditions favourable for the continuous existence of $O$ II in any comparative quantity are absent in many planetaries. In all the planetary nebulæ the governing factor is undoubtedly the surface temperature and physical condition of the nuclear stars. Thirty of these stars are bright enough to yield a spectrum, and all these show the characteristic extension into the extreme ultra-violet denoting $O$ type stars. Half of them contain bright bands, the other half are absorption spectra. There seems, however, to be no correlation between these divisions and the presence or absence of $\lambda 3727$.

Low Wood, Harborne.

\section{The Affinity of Different Types of Enzyme for their Substrates.}

IT is well known that as the concentration of sub. strate molecules is increased, other conditions being kept constant, the rate of catalysis of a reaction by an enzyme reaches a maximum value. On the view that the enzyme-substrate complex is a chemical compound, this is due to the combination of every enzyme molecule with the substrate or its products; on the theory that the union is adsorptive, it is due to saturation of the enzyme surface. On either hypothesis the substrate concentration at which half the maximum velocity is reached furnishes a measure of the affinity between enzyme and substrate. If, as is often the case, the law of mass action is followed, it is, of course, the dissociation constant of the enzyme-substrate compound, and the reciprocal of the affinity constant.

I have collected from the literature such apparent dissociation constants for 44 enzymic reactions. When these are expressed in molar concentrations, the enzymes fall fairly sharply into three groups. Group 1, of low affinity, includes the hydrolytic enzymes acting on crystalloidal substrates, and also yeast carboxylase and liver catalase. Ten of these enzymes, catalysing 21 different reactions, give dissociation constants ranging from $0.003 \mathrm{M}$ up to at least $0.7 \mathrm{M}$. Their affinities for substances related to their substrates which inhibit the reactions are of the same order of magnitude, but on the whole less. Two hydrolytic enzymes, bone phosphatase and liver lipase, yield values at present undetermined, but less than $0.003 \mathrm{M}$ and $0.005 \mathrm{M}$ respectively.

Group 2, of medium affinity, consists of the enzymes which hydrolyse colloidal substrates, namely, the proteins and higher polysaccharides. The rather dubious dissociation constants for these substances mostly, if not all, lie between 5 per cent. and 0.1 per cent., or, taking probable molecular weights, in the neighbourhood of $10^{-4} \mathrm{M}$.

Group 3, of high affinity, consists of the only oxidising-reducing enzymes other than catalase so far studied from this point of view. The values are :

Plant peroxidase . $.5 \times 10^{-6} \mathrm{M}$ [Willstätter \& Weber ${ }^{1}$ ]. Milk xanthine-oxidase $<3 \times 10^{-5} \mathrm{M}$ [Dixon and Thurlow ${ }^{2}$ ]. Yeast oxygenase. $<6 \times 10^{-7} \mathrm{M}$ [Warburg ${ }^{3}$ ].

The substrates were hydrogen peroxide, xanthine or hypoxanthine, and oxygen, respectively. It should be added that xanthine oxidase also slowly catalyses the oxidation of acetaldehyde, but in this case the apparent dissociation constant is of the order of $1 \mathrm{M}$. In the case of laccase, the concentrations of guaiacol giving half the maximum velocity vary with $p H$ between about $0 \cdot 1 \mathrm{M}$ and $0.003 \mathrm{M}$ [Fleury ${ }^{4}$ ]. But laccase is probably a mixture of enzymes including peroxidase, in which case Willstätter and Weber's results make it unlikely that such observations furnish a measure of enzyme-substrate affinity. Biological surfaces which act as catalysts of oxidation, but cannot be brought into colloidal solution, and are, therefore, probably best not included under the designation of enzymes, yield apparent dissociation constants covering a wide range.

While the moderately high affinities of the amylases and proteases may be regarded as an adaptation to the colloidal nature of their substrates, it seems likely that the very high affinity of the oxidases is due to a real difference in the mode of their union with their substrates and that of other enzymes.

$$
\text { J. B. S. HALDANE. }
$$

Dunn Biochemical Laboratory, Cambridge.

\section{Pleochroic Haloes and the Age of the Earth.}

DR. Lotze offers the suggestion, in NATuRE of Jan. 2l, that the effect of the alpha ray in promoting, according to Mügge, "isotropy in minerals, loosening of the crystal lattice, and alterations in cohesion," would account for the unexpectedly large radius of the inner ring of palæozoic uranium haloes.

The stopping power, as all know, is dependent upon the atomic weight of the atoms encountered (Bragg and Kleeman). The density of the medium is therefore involved; and I assume it is to changes in this, brought about by the rays themselves, that Dr. Lotze refers his suggested explanation.

Now the observed discordance of the radius of the inner halo ring with the ionisation curve for uranium and its derivatives is more than 10 per cent. of the estimated radius (Phil. Trans., 217, pp. 51-79). Can the changes which Dr. Lotze postulates be responsible for so great a reduction of density? It seems very improbable. If this considerable loss of density existed, we would expect some optical indications of its existence. But nothing abnormal, compared with the other halo rings, is visible.

1 Willstätter and Weber, Ann. Chem., 449, p. 156; 1926.

2 Dixon and Thurlow, Biochem. Jour., 18, p. 976; 1924.

3 Warburg, Biochem. Zeit., 189, p. 354 ; 1927.

- Fleury, Jour. de Pharm. et Chim., 8, p. $105 ; 1925$.

No. 3041, VoL. 121] 